\title{
BMJ Open Knowledge and attitudes of UK university students in relation to ultraviolet radiation (UVR) exposure and their sun-related behaviours: a qualitative study
}

\author{
Lucy Kirk, ${ }^{1}$ Sheila Greenfield ${ }^{2}$
}

To cite: Kirk L, Greenfield S. Knowledge and attitudes of UK university students in relation to ultraviolet radiation (UVR) exposure and their sun-related behaviours: a qualitative study. BMJ Open 2017;7:e014388. doi:10.1136/bmjopen-2016014388

- Prepublication history and additional material is available. To view please visit the journal (http://dx.doi.org/ 10.1136/bmjopen-2016014388).

Received 20 September 2016 Revised 23 January 2017 Accepted 13 February 2017

CrossMark

\begin{abstract}
${ }^{1}$ College of Medical and Dental Sciences, Birmingham, UK ${ }^{2}$ Institute of Applied Health Research, University of Birmingham, Birmingham, UK
\end{abstract}

Correspondence to Professor Sheila Greenfield; s.m.greenfield@bham.ac.uk

\section{ABSTRACT}

Objectives: To explore whether knowledge about the harms of ultraviolet radiation (UVR) influences UK university students' sun-related behaviours and examine in depth their attitudes towards: sun protection, natural and artificial tanning behaviours.

Design: Qualitative methodology with 15 semistructured, individual interviews. Thematic analysis using the Framework Method with analyst triangulation and member validation.

Setting: One university in the West Midlands, UK. Participants: 15 Caucasian male $(n=4)$ and female $(\mathrm{n}=11)$ students, aged 18-22 years, from a UK university. Participants were recruited using convenience sampling from the university's main campus followed by purposive sampling for: gender, course and sun-related behaviours.

Results: Five main themes emerged: (1) knowledge of UVR; (2) sun-protection practices; (3) attitudes towards tanning; (4) external influences and (5) internal influences. All students knew the associated skin cancer risks from the sun and sunbed use, but this did not appear an important influence in their sun-related behaviours. Body image strongly motivated sunprotection practices and the desire to tan naturally or artificially, across both genders. However, participants' final decision-making appeared to be influenced by their beliefs that practising known harmful sun-related behaviours would not affect them or the perceived susceptibility to sunburn. Beliefs about sunbathing and sunscreen use prompted improper use of sun protection and inadvertently caused more harmful practices. Participants' peers, family and the media had dual roles influencing the development of attitudes towards sun protection and tanning, which contributed to how participants behaved in the sun and their engagement in tanning methods.

Conclusions: Knowledge about the risk of skin cancer associated with UVR did not strongly influence sun-related behaviours, whereas body image appeared as a key motivator. Attitudes towards sun protection and tanning stemmed from the media, peers and family, and particularly from childhood

\section{Strengths and limitations of this study}

- This is the first qualitative study, as far as the authors are aware, that has explored in depth the knowledge and attitudes of students from a UK university towards sun-related behaviours including: sun protection, natural and artificial tanning methods.

- Analyst triangulation was used alongside member validation to corroborate data analysis and strengthen the study's credibility.

- Recruitment difficulties meant that participants were predominately women, therefore attitudes of men who used artificial tanning methods could not be explored.

- Awareness of the interviewer's academic context as a medical student may have encouraged participants to distort their responses towards healthier sun-related attitudes to minimise critical judgement.

habits. Public health strategies may benefit from appearance-related skin cancer prevention campaigns, encouraging safer parental sun-related habits and correcting sun-related misconceptions.

\section{BACKGROUND}

Malignant melanoma is the fifth most common cancer in the $\mathrm{UK}^{1}$ and second most common between 15 and 34 years. $^{2}$ The UK Department of Health ${ }^{3}$ has funded prevention campaigns to address skin cancer, including SunSmart launched in 2003. ${ }^{3} 4$ Despite these efforts, the incidence of melanoma has risen dramatically by more than four times $(360 \%)$ since the late $1970 \mathrm{~s},{ }^{1}$ exceeding growth rates of the ten most common cancers. ${ }^{1} \quad 3 \quad 4$ Consequently, National health Service (NHS) skin cancer 
costs are predicted to increase from £106.4million to $£ 190.5$ million by $2020 .^{5}$

Ultraviolet radiation (UVR) is an environmental risk factor estimated to cause $86 \%$ of skin cancer cases. ${ }^{6}$ With greater accessibility to go abroad and use sunbeds, the rise of melanoma has been largely attributed to changes in sun-related behaviours among the general public, increasing their exposure to higher levels of UVR. ${ }^{7}$ Such changes include: (1) poor sun protection, ${ }^{8}$ (2) sunbathing ${ }^{9}$ and (3) sunbed use, ${ }^{9}$ classified a class 1 carcinogen by the WHO in 2009. ${ }^{10}$ Damage from UVR exposure experienced at younger ages significantly increases the relative risk of skin cancer later in life $;^{11}$ particularly sunburn between 15 and 20 years. ${ }^{7} 12$ However, the popularity of tanning, primarily among the young adult population, further promotes these unsafe sun-related behaviours. ${ }^{12-14}$ Skin cancer prevention therefore remains a public health priority ${ }^{4}$ and should principally target young adults, specifically 16-24 year olds, according to Cancer Research UK. ${ }^{15}$ Yet, this population is less receptive to health education. ${ }^{716}$

For many adolescents, university is the start of their life as a young adult. Parental influences and previous protective barriers can weaken as self-choice and more preeminent influences may determine their behaviours. ${ }^{17}$ Exploration of the knowledge and attitudes that influence UK university students' sun-related behaviours, as a sector of the young adult population, may help identify fundamental areas to be addressed for skin cancer prevention. However, current literature is inconclusive on knowledge influencing individuals' sun-related behaviours, ${ }^{4}{ }^{16-19}$ with many studies also not relevant to UK populations or specific to young adults. Existing evidence on attitudes demonstrates young adults' focus on improving appearance through a tan, with protective behaviours often averted. ${ }^{7}{ }^{16}{ }^{19-21}$ However, studies have yet to qualitatively explore this area pertinent to UK university students, men or those who are not avid tanners. ${ }^{7} 161921$

While women have higher incidence rates of melanoma, ${ }^{1}$ mortality rates are significantly higher in men, ${ }^{1}$ therefore skin cancer prevention in both genders is equally important. Most literature is also primarily based on exploring attitudes towards harmful tanning behaviours, including sunbed use and sunbathing. ${ }^{17} 1921$ There is less evidence about attitudes towards safe artificial practices, namely fake tan; these latter practices may be equally beneficial to explore for promoting healthier sun-related behaviours. All ethnicities are susceptible to skin cancer, however, Caucasians have a significantly higher baseline risk and are often regarded a more concerning population to address. ${ }^{1}{ }^{13}{ }^{19}$ In view of this, a recent qualitative study explored the knowledge and cultural attitudes to sunlight and vitamin D among Caucasian and South Asian focus groups in Greater Manchester. ${ }^{22}$ While it appeared the Caucasian participants had a substantially greater understanding of the risks from sun exposure, the sample age range extended to include much older participants (24-60 years). ${ }^{22}$
This study aims to explore whether knowledge of the harms of UVR influences UK Caucasian University of Birmingham students' sun-related behaviours (1) sun protection; (2) natural tanning (sunbathing); (3) artificial tanning via sunbeds and (4) non-UVR methods (fake $\tan$ ), while simultaneously exploring their attitudes towards them. This may help identify key areas to be addressed for collectively developing effective skin cancer prevention campaigns and promoting healthier sun-related practices among this sector of the young adult population in the UK.

\section{METHODS}

\section{Setting and sample}

The study was conducted at the University of Birmingham, West Midlands where there is an undergraduate and postgraduate student population of $\sim 26500$ (excluding overseas students). ${ }^{23}$ Recruitment started through convenience sampling ${ }^{24}$ to obtain students who were: (1) studying at the University of Birmingham; (2) UK residents; (2) Caucasian and (3) aged 18-24. There were 350 information leaflets, about the study, distributed by hand throughout the main campus and placed on student notice boards throughout the university. Responders were emailed an informed consent form and an eligibility questionnaire (see online supplementary file 1) to then purposively sample $^{24}$ eligible students based on their: sun-related behaviours, current degree course and gender. Owing to recruitment difficulties, nine participants were purposively sampled ${ }^{24}$ and the remaining eligible participants were selected via convenience sampling. ${ }^{24}$

From the 350 leaflets distributed, 27 students responded. Nine did not follow-up and two were not from the UK, therefore not eligible. From the remaining 16 eligible students, one was not required for interview (figure 1). The resultant sample size of 15 was in accordance with Baker and Edwards's ${ }^{25}$ review of sample size in qualitative literature, evidencing that it was a sufficient quantity to achieve data saturation.

\section{Data collection}

Data was obtained through 15 individual, semistructured, audio-recorded interviews in a private room at the University of Birmingham Medical School during February and March 2016, at a time convenient to each participant: no repeat interviews were carried out. Interviews ranged between 23 and $47 \mathrm{~min}$ and were conducted by the same interviewer (LK, a medical student, reading a BMedSc Public Health and Population Science intercalation degree that included course content on qualitative research methodology), with participants aware of the interviewer's characteristics and research rationale. Prior to study initiation, the interviewer was familiar with two of the participants through reading Medicine, but no other relationship had been established with other participants. Written consent was obtained 


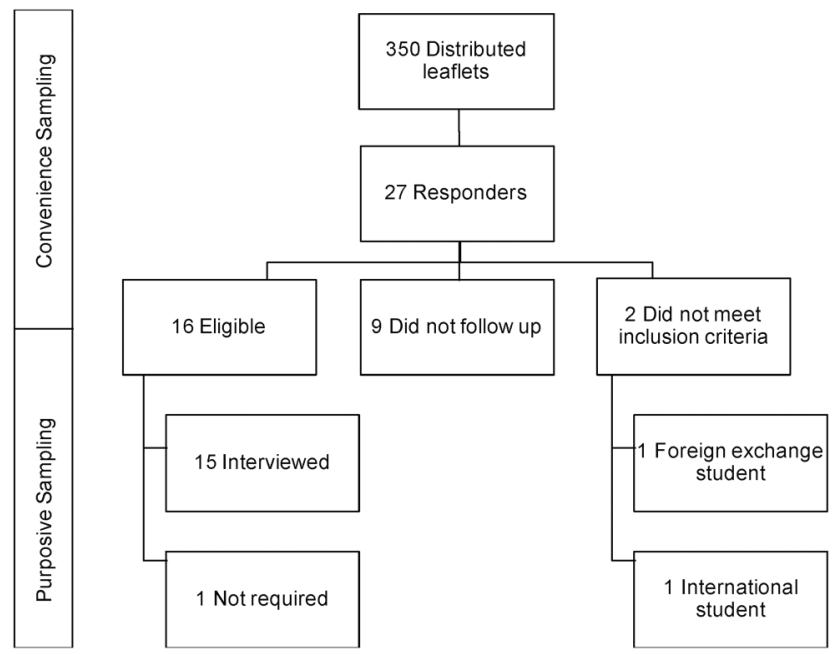

Figure 1 Flow diagram of the participant recruitment process. Responders were students who contacted the researcher to enquire about study participation in response to the leaflets. Students who did not follow-up, were those who did not send back the eligibility questionnaire or did not confirm interest for study participation. One participant was not required following data saturation achievement.

before starting each interview and no non-participants were present. Using relevant literature,,$^{71}{ }^{19-21}$ a topic guide (figure 2) was generated before data collection to provide guidance for answering the primary research aims through use of open-ended questions exploring five key areas: knowledge about UVR, the importance of being tanned, attitudes towards sun protection, natural and artificial tanning behaviours. After each interview, participants received a $£ 15$ Amazon voucher in appreciation of their time.

\section{Data analysis}

Following each interview, field notes were taken to: (1) record emerging themes; (2) reflect on how the interviewer's disciplinary paradigm ${ }^{26}$ as a medical student may have impacted on data collection and (3) contextualise each interview.

Data was then thematically analysed using the Framework Method ${ }^{27}$ as this method is most commonly used for semistructured interview transcripts. ${ }^{28}$ Through use of this method, the audio recordings and subsequent transcripts served as the data to primarily analyse. Further analysis came from the relationship and comparisons made between the primary analyses in order to seek conclusions about the data collectively. ${ }^{28}$ This analysis process occurred through use of several key stages integral to the Framework Method: transcribing, familiarisation, coding, charting and interpretation. ${ }^{27} 28$

First, the audio recordings were transcribed verbatim and listened to twice to ensure transcripts were correct and continue the emergence of themes. By 15 interviews, no more emerging themes were recorded. Two transcripts were randomly selected for independent coding by an additional analyst in analyst triangulation. ${ }^{29}$ This involved familiarisation via thorough slow reading of the data, followed by open coding where transcript content was highlighted and allocated descriptive labels (codes) to describe the concepts and phenomena of the identified text. ${ }^{28}$ Codes and theme development were led entirely by the data, by hand.

Codes were inserted onto a Microsoft Excel spreadsheet per participant. The researcher created preliminary categories, by clustering similar codes developed from the two transcripts and field notes taken during data collection and transcribing. ${ }^{28}$ The researcher and additional analyst met to discuss their analyses and agreed on their identified codes. Both had produced similar codes and concepts from the two transcripts, so mutually agreed the categories created. Categories continued to be developed until all transcripts had been coded and inserted onto the coding spreadsheet. Following 15 transcripts, no new categories had been produced, confirming that data saturation had been met. $^{30}$

Categories were grouped into subcategories and further linked to create themes: together these were used to create the framework matrix that participants' quotes were inserted into, corresponding to their representative subcategory in a process known as charting. ${ }^{27} 28$ Charting provided visual representation of the themes to enable mapping and interpretation of the overall collected data. 27 Member validation further corroborated data analysis, ${ }^{31}{ }^{32}$ by sending a short summary of the overall themes to the participants via email. Three participants responded and stated they were a correct interpretation of their viewpoints. All interviews were included in the analysis and write-up to minimise researcher bias in the selection of quotes used.

To preserve participants' anonymity and confidentiality, numerical pseudonyms were used for data collection and analysis. Gender-specific pseudonyms and participants' ages have been used in the presentation of quotes.

\section{RESULTS}

15 male $(\mathrm{n}=4)$ and female $(\mathrm{n}=11)$ students aged between 18 and 22 were interviewed. Participants' sunrelated behaviours and course characteristics were varied (table 1). A total of $80 \%$ stated a preference for being tanned, 53\% tried to tan and use sun protection, 53\% used non-UVR tanning methods (fake tan) and 20\% had used sunbeds. Participants' degree courses were: medicine $(n=3)$, science and mathematics $(n=3)$, humanities $(\mathrm{n}=7)$ and law $(\mathrm{n}=2)$.

Five main themes, each with subcategories, emerged from the data: knowledge of UVR; sun protection practices; attitudes towards tanning; external influences and internal influences. The themes identify key attitudes and content discussed during the participant interviews that appeared to influence their sun-related behaviours. In contrast, knowledge of UVR developed as a separate 
Figure 2 Topic guide used for participants' interviews. Owing to the nature of semistructured interviews, the exact wording changed depending on the context and the sun-related behaviours stated from the eligibility questionnaire.

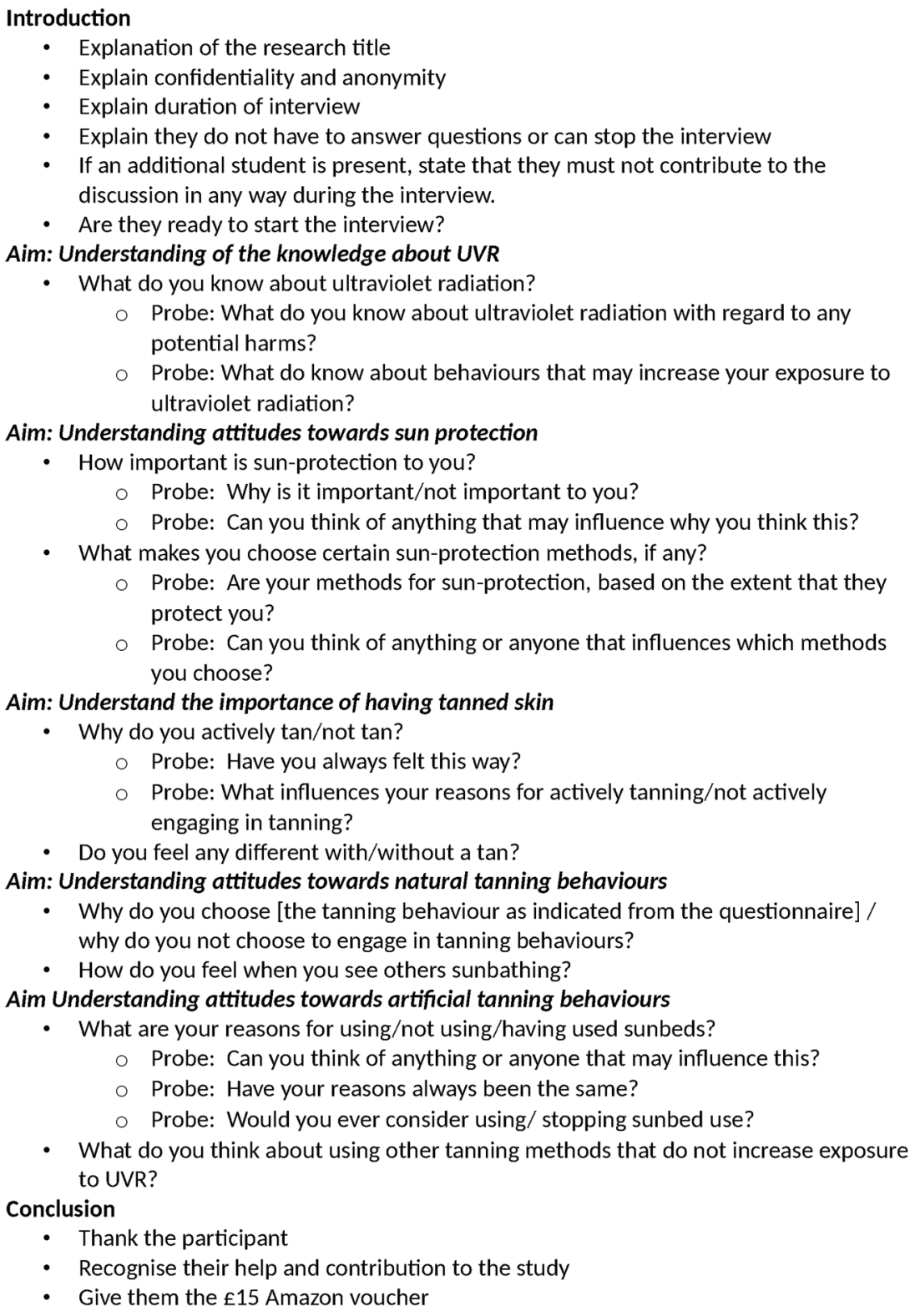

Introduction

- Explain duration of interview

- Explain they do not have to answer questions or can stop the interview

If an additional student is present, state that they must not contribute to the

: Understanding of the knowledge about UVR

at do you know about ultraviolet radiation? potential harms? ultraviolet radiation?

im: Understanding attitudes towards sun protection

important is sun-protection to you?

Probe: Why is it important/not important to you?

Probe: Can you think of anything that may influence why you think this?

- Probe: Are your methods for sun-protection, based on the extent that they protect you? you choose?

m: Understand the importance of having tanned skin

- Probe: What influences your reasons for actively tanning/not actively

m: Understanding attitudes towards natural tanning behaviours

why do you not choose to engage in tanning behaviours?

- What are your reasons for using/not using/having used sunbeds?

- Probe: Can you think of anything or anyone that may influence this?

Probe: Have your reasons always been the same? to UVR?

- Thank the participant

- Give them the 115 Amazon voucher

theme because it did not appear to directly influence participants' sun-related practices nor did the codes and categories included, specifically describe participants' attitudes towards sun behaviours.

Participants' verbatim quotes below reflect each theme and their respective subcategories, with table 2 summarising the charted data.

\section{Knowledge of UVR}

\section{Carcinogenic knowledge}

All 15 participants knew that UVR from sun exposure or sunbed use was associated with the risk of skin cancer. A key difference in knowledge between medical students and those from non-healthcare-related degrees was their molecular understanding of UVR on the skin. However, the concept of skin mutations was not limited to this area of study:
It can affect your cells and it can mutate the DNA, which is something that can cause skin cancers. (Robert, 20)

\section{Public awareness}

Despite information in the public domain relating to the health risks posed by sun-related behaviours, some participants expressed concern that more needed to be done to encourage safer practices, particularly for sunbeds. A direct comparison to the public health campaigns surrounding smoking and lung cancer was occasionally referred to when participants also noted the ease of avoiding health information displayed about skin cancer prevention:

[Information] is quite easily avoided, like if you don't read the back of the bottle you might not know why you're putting sun cream on, [...] whereas if you're a smoker, eg, it's right on the smoking packet, a picture of lung cancer. (Emma, 22) 
Table 1 Participants' characteristics

\begin{tabular}{|c|c|c|c|c|c|c|c|c|c|c|c|c|c|c|c|c|}
\hline \multirow[b]{2}{*}{ Characteristics } & \multicolumn{15}{|c|}{ Participants } & \multirow[b]{2}{*}{ N (\%) } \\
\hline & 1 & 2 & 3 & 4 & 5 & 6 & 7 & 8 & 9 & 10 & 11 & 12 & 13 & 14 & 15 & \\
\hline Age & 22 & 19 & 20 & 20 & 19 & 19 & 20 & 21 & 21 & 19 & 20 & 21 & 22 & 18 & 20 & $18-22$ \\
\hline \multicolumn{17}{|l|}{ Gender } \\
\hline Male & $\checkmark$ & $\checkmark$ & & & & & & $\checkmark$ & & & & $\checkmark$ & & & & $4(27)$ \\
\hline Female & & & $\checkmark$ & $\checkmark$ & $\checkmark$ & $\checkmark$ & $\checkmark$ & & $\checkmark$ & $\checkmark$ & $\checkmark$ & & $\checkmark$ & $\checkmark$ & $\checkmark$ & $11(73)$ \\
\hline \multicolumn{17}{|l|}{ Courses } \\
\hline Medicine & & & & & & & & & $\checkmark$ & $\checkmark$ & & & $\checkmark$ & & & $3(20)$ \\
\hline Humanities & $\checkmark$ & $\checkmark$ & & & & $\checkmark$ & $\checkmark$ & $\checkmark$ & & & $\checkmark$ & $\checkmark$ & & & & $7(47)$ \\
\hline Science and maths & & & & $\checkmark$ & & & & & & & & & & $\checkmark$ & $\checkmark$ & $3(20)$ \\
\hline Law & & & $\checkmark$ & & $\checkmark$ & & & & & & & & & & & $2(13)$ \\
\hline \multicolumn{17}{|l|}{ Preference for being tanned } \\
\hline Yes & $\checkmark$ & $\checkmark$ & $\checkmark$ & $\checkmark$ & $\checkmark$ & $\checkmark$ & $\checkmark$ & & $\checkmark$ & & $\checkmark$ & $\checkmark$ & $\checkmark$ & & $\checkmark$ & $12(80)$ \\
\hline No & & & & & & & & & & $\checkmark$ & & & & & & $1(7)$ \\
\hline No preference & & & & & & & & $\checkmark$ & & & & & & $\checkmark$ & & $2(13)$ \\
\hline \multicolumn{17}{|l|}{ Behaviour in the sun } \\
\hline Tan as much as possible & & & & & $\checkmark$ & & $\checkmark$ & & $\checkmark$ & & & & & & $\checkmark$ & $4(27)$ \\
\hline Tan and use sun protection & & $\checkmark$ & $\checkmark$ & $\checkmark$ & & $\checkmark$ & & & & & $\checkmark$ & $\checkmark$ & $\checkmark$ & $\checkmark$ & & $8(53)$ \\
\hline Protect as much as possible & $\checkmark$ & & & & & & & & & $\checkmark$ & & & & & & $2(13)$ \\
\hline No preference & & & & & & & & $\checkmark$ & & & & & & & & $1(7)$ \\
\hline \multicolumn{17}{|l|}{ Artificial tanning behaviour } \\
\hline Sunbed use & & & & & & & $\checkmark$ & & $\checkmark$ & & & & $\checkmark$ & & & $3(20)$ \\
\hline Non-UVR tanning & & & $\checkmark$ & $\checkmark$ & $\checkmark$ & $\checkmark$ & $\checkmark$ & & $\checkmark$ & & $\checkmark$ & & $\checkmark$ & & & $8(53)$ \\
\hline No use of artificial tanning & $\checkmark$ & $\checkmark$ & & & & & & $\checkmark$ & & $\checkmark$ & & $\checkmark$ & & $\checkmark$ & $\checkmark$ & $7(47)$ \\
\hline
\end{tabular}

\section{Sun protection practices}

\section{Short-term damage}

Sun protection centred on sunscreen. Additional methods, such as seeking shade or covering up were less frequently referred to; here, this was mostly in response to sunburn. While all participants acknowledged the importance of sun protection, motivating factors primarily focused on short-term prevention of sunburn and sunstroke. Main concerns were the physical manifestations of sunburn: pain, appearance and reduced activity:

I don't want like a really red face, or you know, skin that like hurts to touch and stuff like that. (Michael, 19).

However, one participant positively associated sunburn with tanning:

I'm probably happy that I've sunburnt a little bit because I know that I'll get a bit of a colour. (Elizabeth, 20)

Some participants did consider the long-term damages, namely skin cancer and ageing. This mostly presented in response to sunburn, serving as a visible reminder for sun protection:

It's quite obvious that you've damaged your skin when you've got burnt and it makes me worry a bit about how much damage I have caused to myself. (Sophie, 20)

\section{Sun protection drawbacks}

Drawbacks of sun protection were evident among participants. For sunscreen, this included cost and time required for application, and its smell, sensation and appearance once applied onto skin. Consequently, sun protection was regarded a 'necessary evil' (Robert, 20) with the negative qualities of sunscreen presenting as occasional barriers for its use:

It smells, and if you're on the beach you get sand stuck to you, it's just a pain, and so then it's only when it actually probably definitely needs doing that I will do it. (Jack, 22)

\section{Being abroad}

Participants predominately considered sun protection when abroad, compared with in England. Regardless of summer, sun protection was used as part of a holiday mentality with the expectation of being in a hotter and brighter environment. This resulted in unexpected sunburn in England as expressed by three participants:

In the UK if I go out and it's a summer's day, I won't always bring sun cream with me because it's just not really on my mind then. (Amy, 20)

\section{Attitudes towards tanning}

Self-image

For all participants with a preference for tanning, this was rooted in self-image. A tan offered aesthetic 


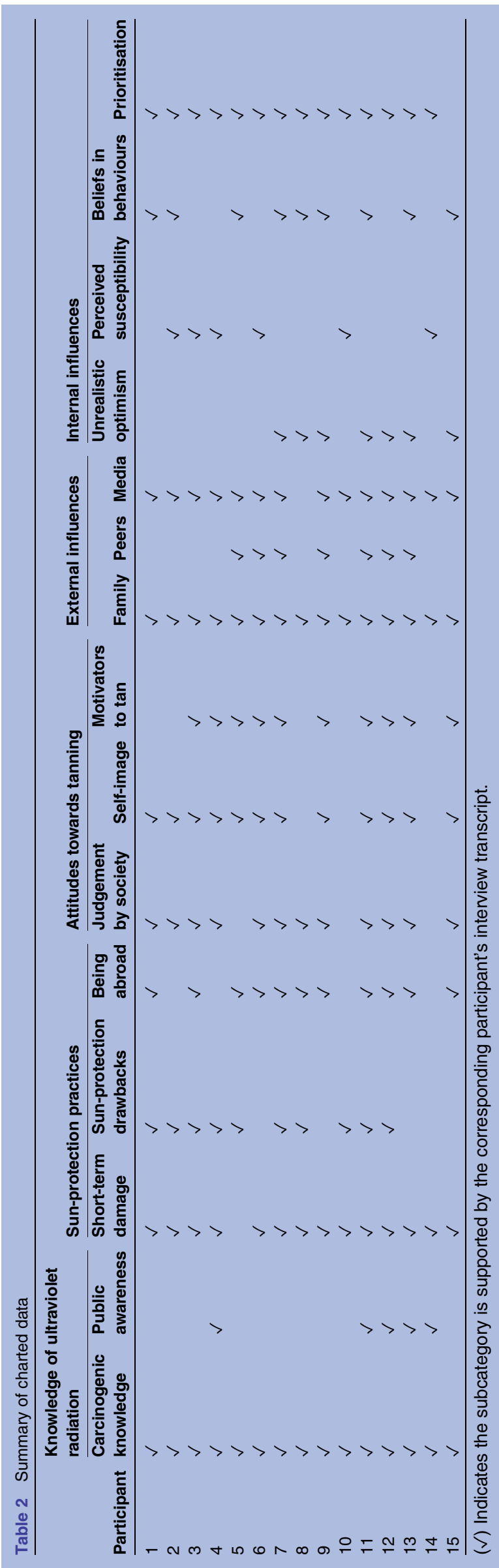

enhancements generally and specifically: looking slimmer, covering scars, improving skin complexion and having a 'healthy glow' (Holly, 19). This resulted in psycho-sociological benefits from a tan including improved social confidence and a sense of health and well-being:

Tanning gives [you] more of like a slimmer look as well. And obviously if you feel like you look good, you will feel good. You'll have a bit more confidence. (Holly, 19).

Caveats to this reflected concerns on the concept of 'orange' (Harriet, 20) or 'patchy' (Rebecca, 19) results gained from fake tanning products limiting their authenticity over sun or sunbed achieved colour. Clear associations were also made about a tan; it served as a positive reminder of leisure, holidays and health. While a tan was associated with freedom and relaxation, deep tans were less viewed as healthy:

There's extremes to this. I mean you see people on Geordie Shore and TOWIE [...] and they're extremely tanned. Sunbeds everyday. But I think a bit of a tan does make you look healthier. (Natasha,21).

\section{Judgement by society}

Many participants commented on skin colour within the context of how they are perceived by others in society and among their social groups. A tan represented a good and healthy lifestyle, ability to travel and wealth: 'it suggests you can afford to go away' (Sophie, 20). Reinforcing these concepts, were the positive comments received by the participants in relation to being tanned. Conversely, being pale provoked feelings of insecurity and concerns about what others might be thinking:

If I'm like topless and I'm pale and gardening, I'm aware someone might look at me and say or just think that 'he doesn't get outside much'. (Robert, 21).

Irrespective of their preference for tanning, there was a subliminal awareness of the pressure to have one, particularly following a holiday and during the summer:

[when] it's summer, it's a good thing to get a tan. That's what you're meant to do. It's not, like, 'stay pale'. (Alistair,21).

This social constraint was sometimes questioned by participants on exploration for their preference for being tanned:

I do like having a tan, but it's always hard to tell how much you actually think it and whether you have just like, been almost brought up to believe that it looks good. (Michael, 19).

\section{Motivators to tan}

During summer months, tanning was regarded a natural process and by-product of enjoying the warmth from the 
sun; consequently, in winter, it was viewed unnecessary. Exceptions to this seasonal preference were with women who engaged in artificial tanning methods before special occasions and social events. Provokers for sunbed use also included 'base-tan' preparation before a holiday to minimise sunburn while tanning:

If I was going on holiday and hadn't seen the sun in a while, I might go on a few [sunbeds] before then so that I didn't burn when I got on holiday. (Emma, 22).

\section{External influences}

Family

The childhood experiences of all participants moulded their views on the importance of using sun protection. Practices learnt early on influenced decision-making as adults on safer sun-related behaviours and the adoption of sun-protective routines-all based on awareness of the harms of UVR. Conversely, family also played a role in the practice of harmful behaviours. Sibling competitiveness to achieve a darker tan, parental compliments and tanning behaviours equally motivated the desire to actively tan and reduced sun-protection vigilance:

We all get sunburnt at the same time [...] like we all want to get suntanned but we don't really think about the health risk. (Charlotte, 19).

\section{Media}

The media had two distinctive roles. Promoting safer sun-related practices was through education on harmful behaviours and increasing awareness about skin cancer through television documentaries, magazine spreads, news reports and advertisements. Equally, the cult of celebrities and media role-models inspired the desire to tan actively and underpinned the pressure to be tanned:

Usually on like TV and adverts and stuff they'll have women who are tanned and they won't really have pale women. (Olivia,18).

\section{Peers}

Collective behaviours of participants equally influenced tanning behaviours where sunbathing, sunbed use and application of fake tan were part of their peer group activities:

[on holiday] all my friends are tanning [so] I just kind of join in (Harriet,20).

Competitiveness among friends to achieve a natural tan on holiday was recognised by five participants. Protective behaviours were also influenced by peers. While four participants stated they actively try to encourage protective practices among their peers, many felt more easily distracted by their friends and less vigilant about sun protection in the absence of their parents:
My parents would encourage me like 'be careful' whereas your friends, you're kind of looking more out for yourself, so you probably wouldn't bother so much. (Katie,19)

\section{Internal influences}

Unrealistic optimism

Many participants disregarded their knowledge on the harms of UVR in the self-reassurance that these would not affect them or represented an imminent threat. This unrealistic optimism ${ }^{33}$ appeared to be a fundamental factor governing participants' decisions to engage in risky sun-related behaviours.

I know it's damaging but at the same time it's just like $[\ldots]$ I just assume it would never affect me kind of thing. (Alistair,21)

\section{Beliefs in behaviours}

Beliefs that tanning was the skin adapting to UVR exposure and was protective against sunburn, prompted the idea of being able to 'sunbathe safely', therefore gradually lowering levels of sun protection. It was also the rationale for a 'base-tan' achieved from a sunbed, despite participants' knowledge on the harms:

I'm always wanting to [tan] in the safest way possible and minimum harm to the skin. So I'll wear like factor 50, the first couple of days and gradually go down as time goes on. (Holly,19)

\section{Perceived susceptibility}

Personal experiences, primarily extreme sunburn, frequently motivated participants to use sun protection through a heightened awareness for their skin's susceptibility to sun damage. Perceived susceptibility played a key role in decisions not to engage in UVR exposure. Those with greater risk of sunburn were more concerned with protection regardless of their preference for tanning:

I feel like I don't really tan so [I] just probably [try] to protect myself, but I don't know if it'd be different if I was like the kind of person who'd tan. (Katie,19)

While knowledge of someone with skin cancer increased participants' awareness, it did not appear to deter them away from actively sunbathing.

\section{Prioritisation}

All participants expressed health as a priority over skin damage and tanning, echoed by preferences for fake tan over sunbeds and use of sun protection. However, with the exception of those who expressed inability to tan, prioritising health in the sun was considerably less than initially suggested with tanning motives preventing maximum sun protection: 
I think normally I will consciously start off with like a lower sort of level of sunscreen in order to try and tan more. (Jack,22)

Yet, for five participants, including two sunbed users, facial protection was consistently prioritised over tanning through concerns of ageing. Prioritising money and time, also governed decisions, in particular sunbed use regardless of its health implications. With fake tan, decisions related to affordability and the process involved:

I know the health issues [of sunbeds], but mainly because of time, price. I just have other hobbies I'd like to invest my time and money in. (Jack,22)

Themes and their respective subcategories are summarised in figure 3 which demonstrates the close relationship and complexity of links between them.

\section{DISCUSSION}

\section{Main findings}

This study explored whether knowledge influences sunrelated behaviours and attitudes towards sun protection and tanning among UK university students. From the data, five themes emerged: knowledge of UVR; sun protection practices; attitudes towards tanning; external influences and internal influences. Results highlight the complexity of sun-related behaviours and from a public health context, there appear key areas that could be addressed for skin cancer prevention.

Findings concurred with existing evidence that knowledge of the associated risks from UVR was not sufficient

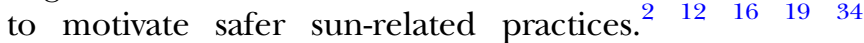
Degree-related UVR knowledge, particularly those studying medicine, also appeared of little influence; similarly found among Swedish university students. ${ }^{35}$ While all participants acknowledged the importance of sun protection, primarily sunscreen, this was frequently limited to being abroad, causing complacency in England. Furthermore, despite incorrect use of sunscreen, its application falsely reassured participants their behaviours were safe. As sunscreen has equally been found to encourage greater sun exposure ${ }^{36} 37$ and is not sufficient protection alone, ${ }^{17}$ 37-39 additional methods of protection, shade and covering up, must be promoted. Participants infrequently equated a tan with sun damage and instead viewed deepening colour with opportunity to lower sunscreen factor without risk of sunburn, thus

Sun-protection
practice was
affected by:
difference in sun-
protection attitudes
between being in
England and being
abroad, drawbacks
of sun-protection
methods and
attitudes towards
the short-term
damages from the
sun

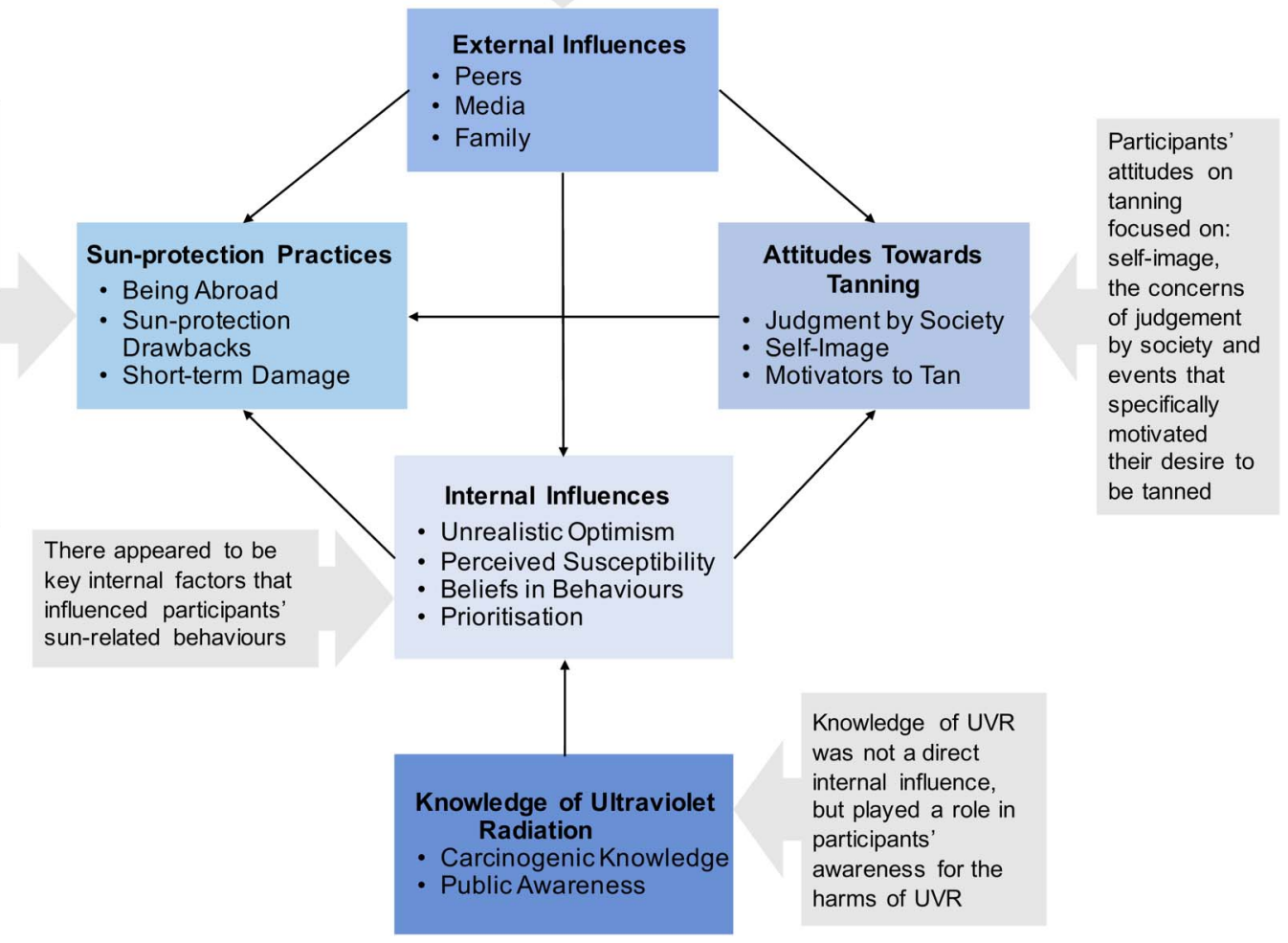

Figure 3 Main theme links. Diagram to illustrate how the main themes interlink together, indicating the complexity of attitudes and influences related to sun-related behaviours that emerged from the data. Each box contains one theme and the respective subcategories. 
'sunbathing safely'. Similar reasons prompted a 'base-tan' from sunbeds before starting a holiday. These false concepts $^{40}$ are reported in a multitude of studies, ${ }^{2}{ }^{19} 41-43$ but, contradictory to Schneider and Kramer's' 2009 review, ${ }^{18}$ participants did know the associated risks from sunbed use: two sunbed users were medical students. Correcting sun protection and tanning misconceptions is imperative.

A notable difference between those who did and did not engage in harmful sun behaviours alluded to unrealistic optimism. ${ }^{33}$ This is widely reported in western literature among all ages of avid tanners, ${ }^{19}{ }^{44-47}$ but this study demonstrates its presence also in those without a preference for tanning. Paradoxically, health risks associated with usage of low factor sun protection were known, but taken as informed ones to enable tanning, while sunbeds were avoided. Conversely, unrealistic optimism was not apparent among participants expressing their skin type as highly susceptible to burning, consistent with Becker's (1984) Health Belief Model ${ }^{48}$ regarding perceived susceptibility. Those who stated an inability to tan regardless of their preference for darker skin, focused on protection, echoing Clarke et al's ${ }^{49}$ study on skin type and sun behaviours.

Media, family and peers strongly influenced attitudes towards harmful and safe sun-related behaviours; this is quantitatively supported by a study of British university students. $^{7}$ In protecting against UVR, family, mainly parents, initiated practices that continued from childhood to young adulthood. In parallel with western literature on young adults' tanning motives, the subliminal message that a tan is a 'good thing', came from family, ${ }^{50}$ alongside peers ${ }^{19}$ and the media, ${ }^{51}$ who all encouraged tanning with appearance-related benefits and as a healthy lifestyle choice, resulting in psychological improvements. ${ }^{17} 19-2140$ Consequently, participants associated pale skin colour with feelings of insecurity, irrespective of gender. Fake tan limitations primarily related to appearance, through its lack of authenticity; but it was a 'quick fix' before social events and was preferred over the health implications of sunbed use. Physical manifestations of burning, namely redness, strongly encouraged sun protection usage, with facial prioritisation prompted by premature ageing concerns-concerns equally reported by Murray and Turner. ${ }^{21}$ Here, findings suggest Slade's (1994) theory on 'body image, ${ }^{52}$ strongly motivated protective and harmful sun-related practices.

Changing society's portrayal of a tan could initiate safer behaviours, but the trend has remained consistent since the 1920s. ${ }^{53}$ In the context of increasing public awareness, the success of pictorial images on cigarette packaging, increasing incentives to quit smoking, ${ }^{54}$ suggests future campaigns adopting images relevant to appearance-related UVR damage may be equally effective and more achievable. Media dissemination could enhance uptake. While appearance-focused prevention is recommended ${ }^{40} 4255$ and has been found more effective than focusing on skin cancer risk, ${ }^{9}$ studies differ as to how best to demonstrate the dangers of UVR. ${ }^{40}$ Graphic images to 'shock' the public were suggested among the study's participants and are favoured by older boys, ${ }^{56}$ yet a study of UK adults argued such images would be inappropriate. ${ }^{45} \mathrm{~A}$ mixed-methods study would prove beneficial to explore the public's opinion, meanwhile fake tan methods as a safer tanning alternative, should be widely promoted. While cost has not been found to deter people from sun protection, ${ }^{4}$ expenses influenced decision-making among participants, particularly relating to higher sunscreen factors and artificial tanning. Consequently, incentives to use sunbeds are promoted via student discount deals within existing tanning salons. The accessibility of sunbeds has shifted the skin cancer demographics of young women to the North West of England. ${ }^{57}$ Previous trends had seen cases in higher socioeconomic groups. ${ }^{57}$

\section{Strengths and limitations}

To the best of our knowledge, this is the first qualitative study exploring the knowledge and attitudes of UK university students in relation to UVR exposure and their sun-related behaviours. Findings highlight key issues that are widely supported among the literature, suggesting likely transferability to other UK university settings. ${ }^{29}$ However, results may not be transferable to nonuniversity students owing to the potential for educative and socioeconomic differences. Study credibility was strengthened by analyst triangulation and member validation that confirmed themes and subcategories were a correct interpretation of participants' viewpoints. ${ }^{29} 31$ Extending analyst triangulation throughout data analysis with multiple analysts and a greater number of member validation responses would, however, have improved trustworthiness. Methods triangulation would have also helped to further corroborate findings. ${ }^{31}$ Given the qualitative nature of the study, its findings do not serve to be generalisable but to provide a more in-depth insight into the complex area of sun-related behaviours on a population that has not yet been explored. While the small sample size of 15 may present as a limitation, it is supported by existing literature exploring the extensive debate on the number of interviews required for qualitative research. However, recruitment difficulties did limit purposive sampling therefore attitudes of male sunbed users could not be explored. Social desirability bias $^{58}$ from the use of individual interviews and participants' awareness that the interviewer was a medical student may have affected truthfulness in the collected data: but with findings widely supported by existing literature, this is likely to have had only a minimal effect.

\section{Research and policy recommendations}

Beliefs related to tanning as a protective phenomenon and ability to sunbathe safely with sunscreen show common misconceptions that need to be addressed at a public health level. A mixed-methods study exploring public opinion and quantifying the effectiveness of using 
pictorial images showing appearance-related effects of UVR exposure would be useful for developing potential novel strategies for skin cancer prevention. With reduced living costs often experienced by university students, changes in the costs of sunscreen and artificial methods may help encourage safer behavioural changes. Equally, childhood habits played a key role in participants' attitudes towards sun protection and tanning. This illustrates the potential need for the promotion of safer sun-related behaviours starting from within the family setting.

\section{CONCLUSION}

For UK university students, the complexity of attitudes towards harmful and safe sun-related behaviours may be primarily influenced by their family, peers and the media. Family, mainly parents, may therefore serve as a potential platform to encourage safer sun-related habits starting from childhood. The role of body image that motivated tanning and sun protection practices suggests appearance-related campaigns for skin cancer prevention could be paramount for effectively promoting safer sun-related behaviours for UK university students. While knowledge about the risks of skin cancer associated with UVR may not appear to strongly affect sun-related behaviours for this population, it remains imperative to correct common sun-related misconceptions.

Acknowledgements We would like to thank all the participants for their contribution to the study and Lucy Milton and Erin Timmins for administrative support.

Contributors LK conceived and designed the study, with guidance from SG who also provided qualitative methodological support. LK conducted and transcribed all the interviews. LK conducted the analysis alongside SG who served as the additional analyst during the coding process and development of subcategories. LK produced the final manuscript under the supervision of SG.

Funding This research received no specific grant from any funding agency in the public, commercial or not-for-profit sectors. SG is part funded by the National Institute for Health Research (NIHR) and Collaboration for Leadership in Applied Health Research and Care (CLAHRC).

Disclaimer The views expressed are those of the authors and not necessarily those of the NIHR, the NHS or the Department of Health.

\section{Competing interests None declared.}

Ethics approval Ethical approval was granted by the BMedSc Population Sciences and Humanities Internal Ethics Review Committee at the University of Birmingham. Data archives will be stored at the University of Birmingham, in accordance with the University's code of practice.

Provenance and peer review Not commissioned; externally peer reviewed.

Data sharing statement No additional data are available.

Open Access This is an Open Access article distributed in accordance with the Creative Commons Attribution Non Commercial (CC BY-NC 4.0) license, which permits others to distribute, remix, adapt, build upon this work noncommercially, and license their derivative works on different terms, provided the original work is properly cited and the use is non-commercial. See: http:// creativecommons.org/licenses/by-nc/4.0/

\section{REFERENCES}

1. Cancer Research UK. Skin cancer incidence statistics. 2015. http:// www.cancerresearchuk.org/health-professional/cancer-statistics/
statistics-by-cancer-type/skin-cancer/incidence\#heading-One\% $2013,300 \% 20$ cases\%20of\%20mm\%20in\%202011\%20diagnosed (accessed Apr 2016).

2. Lake JR, Thomson CS, Twelves CJ, et al. A qualitative investigation of the motivations, experiences and views of female sunbed users under the age of 18 in England. J Public Health (Oxf) 2014;36:56-64.

3. Cancer Research UK. About the SunSmart campaign. 2014. http:// www.cancerresearchuk.org/health-professional/prevention-andawareness/sunsmart/about-the-sunsmart-campaign (accessed Apr 2016).

4. Peacey V, Steptoe A, Sanderman R, et al. Ten-year changes in sun protection behaviours and beliefs of young adults in 13 European countries. Prev Med 2006;43:460-5.

5. Vallejo-Torres L, Morris S, Kinge JM, et al. Measuring current and future cost of skin cancer in England. J Public Health (Oxf) 2014;36:140-8.

6. Parkin DM, Mesher D, Sasieni P. 13. Cancers attributable to solar (ultraviolet) radiation exposure in the UK in 2010. Br J Cancer 2011;105(Suppl 2):S66-9. http://dx.doi.org/ 10.1038/bjc.2011.486

7. Grunfeld EA. What influences university students' intentions to practice safe sun exposure behaviours? J Adolesc Health 2004;35:486-92.

8. Williams $\mathrm{M}$, Caputi $\mathrm{P}$, Jones $\mathrm{SC}$, et al. Sun protecting and sun exposing behaviors: testing their relationship simultaneously with indicators of ultraviolet exposure among adolescents. Photochem Photobiol 2011;87:1179-83.

9. Jones JL, Leary MR. Effects of appearance-based admonitions against sun exposure on tanning intentions in young adults. Health Psychol 1994;13:86-90.

10. El Ghissassi F, Baan R, Straif K, et al. WHO International Agency for Research on Cancer Monograph Working Group: a review of human carcinogens-part D: radiation. Lancet Oncol 2009;10:751-2.

11. Autier P, Dore JF. Influence of sun exposures during childhood and during adulthood on melanoma risk. EPIMEL and EORTC Melanoma Cooperative Group. European Organisation for Research and Treatment of Cancer. Int J Cancer 1998;77:533-7.

12. Stott MA. Tanning and sunburn: knowledge, attitudes and behaviour of people in Great Britain. J Public Health Med 1999;21:377-84.

13. Friedman B, English JC, Ferris LK. Indoor tanning, skin cancer and the young female patient: a review of the literature. J Pediatr Adolesc Gynecol 2015;28:275-83.

14. Diffey B. Sunbeds, beauty and melanoma. Br J Dermatol 2007;157:215-6.

15. Cancer Research UK. Trends in awareness and behaviour relating to UV and sun protection: 2003-2013. 2014. http://www.cancerresearchuk. org/sites/default/files/sun protection trends - cruk.pdf (accessed Apr 2016).

16. Norton E, Holloway I, Galvin K. Comfort vs risk: a grounded theory about female adolescent behaviour in the sun. J Clin Nurs 2014;23:1889-99.

17. Day AK, Wilson CJ, Hutchinson AD, et al. The role of skin cancer knowledge in sun-related behaviours: a systematic review. J Health Psychol 2014;19:1143-62 doi: 10.1177/1359105313485483 [published Online First: 16 May 2013].

18. Schneider S, Krämer H. Who uses sunbeds? A systematic literature review of risk groups in developed countries. J Eur Acad Dermatol Venereol 2010;24:639-48.

19. Dennis LK, Lowe JB, Snetselaar LG. Tanning behaviour among young frequent tanners is related to attitudes and not lack of knowledge about the dangers. J Health Educ 2009;68:232-43.

20. Kyle RG, MacMillan I, Forbat L, et al. Scottish adolescents' sun-related behaviours, tanning attitudes and associations with skin cancer awareness: a cross-sectional study. BMJ Open 2014;4: e005137.

21. Murray CD, Turner E. Health, risk and sunbed use: a qualitative study. Health Risk Soc 2004;6:67-80.

22. Webb AR, Aseem S, Kift RC, et al. Target the message: a qualitative study exploring knowledge and cultural attitudes to sunlight and vitamin D in Greater Manchester. Br J Dermatol 2016;175:1401-1403. http://dx.doi.org/10.1111/bjd.14800

23. Birmingham.ac.uk. Who studies here?-University of Birmingham. 2015. http://www.birmingham.ac.uk/university/fact/who.aspx (accessed Apr 2016).

24. Marshall MN. Sampling for qualitative research. J Fam Practice 1996;13:522-6.

25. Baker S, Edwards R. How many qualitative interviews is enough? ESRC National Centre for Research Methods review paper. http:// eprints.ncrm.ac.uk/2273/4/how_many_interviews.pdf (accessed Nov 2016). 
26. Krefting L. Rigor in qualitative research: the assessment of trustworthiness. Am J Occup Ther 1991;45:214-22.

27. Ritchie J, Spencer E. Qualitative data analysis for applied policy research. In Bryman A, Burgess RG, eds. Analyzing qualitative data. London: Routledge, 1994:173-94.

28. Gale NK, Heath G, Cameron E, et al. Using the framework method for the analysis of qualitative data in multi-disciplinary health research. BMC Med Res Methodol 2013;13:117.

29. Lincoln YS, Guba EG. Naturalistic inquiry. Newbury Park, CA: Sage Publications, 1985

30. Seale C. The quality of qualitative research. London: Sage Publications, 1999.

31. Patton MQ. Enhancing the quality and credibility of qualitative analysis. Health Serv Res 1999;34:1189-208.

32. Walker $\mathrm{S}$, Read S, Priest $\mathrm{H}$. Use of reflexivity in a mixed-methods study. Nurse Res 2013;20:38-43.

33. Weinstein ND. Unrealistic optimism about future life events. $J$ Pers Soc Psychol 1980;39:806-20.

34. Keeney S, McKenna H, Fleming $\mathrm{P}$, et al. Attitudes, knowledge and behaviours with regard to skin cancer: a literature review. Eur J Oncol Nurs 2009;13:29-35.

35. Jerkegren E, Sandrieser L, Brandberg Y, et al. Sun-related behaviour and melanoma awareness among Swedish university students. Eur J Cancer Prev 1999;8:27-34.

36. McGregor JM, Young AR. Sunscreens, suntans, and skin cancer. BMJ 1996;312:1621-2.

37. Autier P, Dore JF, Schifflers E, et al. Melanoma and use of sunscreens: the EORTC Melanoma Co-operative Group. Int $J$ Cancer 1995;61:1749-55.

38. Linos E, Keiser E, Fu T, et al. Hat, shade, long sleeves, or sunscreen? Rethinking US sun protection messages based on their relative effectiveness. Cancer Causes Control 2011;22:1067-71

39. Autier P, Dore JF, Cattaruzza MS, et al. Sunscreen use, wearing clothes, and number of nevi in 6- to 7-year-old European children. European Organization for Research and Treatment of Cancer Melanoma Cooperative Group. J Natl Cancer Inst 1998;90:1873-80.

40. Lim HW, James WD, Rigel DS, et al. Adverse effects of ultraviolet radiation from the use of indoor tanning equipment: time to ban the tan. J Am Acad Dermatol 2011;64:e51-60.

41. Garside R, Pearson M, Moxham T. What influences the uptake of information to prevent skin cancer? A systematic review and synthesis of qualitative research. Health Educ Res 2010;25:162-82.

42. Autier P. Perspectives in melanoma prevention: the case of sunbeds. Eur J Cancer 2004;40:2367-76.

43. Jones F, Harris P, Chrispin C. Catching the sun: an investigation of sun-exposure and skin protective behavior. Psychol Health Med 2000;5:131-41.
44. Lucci A, Citro HW, Wilson L. Assessment of knowledge of melanoma risk factors, prevention, and detection principles in Texas teenagers. J Surg Res 2001;97:179-83.

45. Wright L, Bramwell R. A qualitative study of older people perceptions of skin cancer. Health Educ J 2001;60:256-64

46. Bränström R, Kristjansson S, Ullén H. Risk perception, optimistic bias, and readiness to change sun related behaviour. Eur J Public Health 2006;16:492-7.

47. Monfrecola G, Fabbrocini G, Posteraro G, et al. What do young people think about the dangers of sunbathing, skin cancer and sunbeds? A questionnaire survey among Italians. Photodermatol Photoimmunol Photomed 2000;16:8-15

48. Becker $\mathrm{MH}$. The health belief model and personal health behavior. Health Educ Monogr 1974;2:324-508.

49. Clarke VA, Williams T, Arthey S. Skin type and optimistic bias in relation to the sun protection and suntanning behaviors of young adults. J Behav Med 1997;20:207-22.

50. Stanton WR, Janda M, Baade PD, et al. Primary prevention of skin cancer: a review of sun protection in Australia and internationally. Health Promot Int 2004;19:369-78 doi: 10.1093/heapro/dah310 http://dx.doi.org/10.1093/heapro/dah310

51. NICE. Skin cancer prevention guidelines-expert paper 6 . The impact of role models on sun protective behaviours: expert paper. 2009. https://www.nice.org.uk/guidance/ph32/documents/expertpaper-6-the-impact-of-role-models-on-sun-protection-behaviours2 (accessed Apr 2016).

52. Slade PD. What is body image? Behav Res Ther 1994;32 497-502.

53. Chang C, Murzaku EC, Penn L, et al. More skin, more sun, more tan, more melanoma. Am J Public Health 2014;104:e92-9. http://dx. doi.org/doi: 10.2105/AJPH.2014.302185

54. Borland $\mathrm{R}$, Yong $\mathrm{HH}$, Wilson $\mathrm{N}$, et al. How reactions to cigarette packet health warnings influence quitting: findings from the ITC Four-Country survey. Addiction 2009;104:669-75.

55. Hillhouse JJ, Turrisi R, Kastner M. Modeling tanning salon behavioral tendencies using appearance motivation, self-monitoring and the theory of planned behavior. Health Educ Res 2000;15:405-14.

56. Paul C, Tzelepis F, Girgis A, et al. The slip slop slap years: have they had a lasting impact on today's adolescents? Health Promot $J$ Austr 2003;14:219-21.

57. Wallingford SC, Alston RD, Birch JM, et al. Regional melanoma incidence in England, 1996-2006: reversal of north-south latitude trends among the young female population. $\mathrm{Br} J$ Dermatol 2013;169:880-8.

58. Social Desirability Bias: SAGE Research Methods. 2015. https:// srmo.sagepub.com/view/the-sage-encyclopedia-of-social-scienceresearch-methods/n932.xml (accessed Apr 2016). 